\title{
Metacontrast with increases in the number of masking ring segments
}

\author{
WILLIAM N. DEMBER and DONNA ARAND \\ University of Cincinnati, Cincinnati, Ohio 45221
}

\begin{abstract}
The present experiment replicated and extended earlier studies which examined the effect of increases in the number of masking ring segments on the detectability of a black target disk. It confirmed earlier findings that masking effectiveness increases with increases in black ring segments up to 16 , where asymptote is reached. Beyond this value, the present study demonstrates a decline in masking with increases in black mask segments up to 64 , indicating an inverted U-shaped relationship between number of ring segments and mask effectiveness. The failure of current theories to account for this finding and implications for future theories are discussed.
\end{abstract}

The present study is one in a series of experiments which investigates the effect of configuration variables on metacontrast by applying the forced-choice response methodology to the disk-ring paradigm used by Werner (1935). In particular, the present study extends the results of those experiments investigating the masking effectiveness of masking rings having incomplete contours. An early study by Sherrick and Dember (1968) used masking rings in which $60 \%$ of the black ring contour was removed, while the remaining contour was divided into two, four, six, or eight equal-sized black segments and distributed evenly around the ring (alternated with an equal number of white segments). The results showed that masking effectiveness increased as the number of ring segments increased.

A subsequent study by Dember, Colina, and Sherrick (1972) used two sets of masking rings. In one set $25 \%$, $50 \%$, or $75 \%$ of a complete ring was removed, and the remaining contour was divided into four equal-sized segments and evenly distributed around the ring. A second set of masks consisted of complete gray rings having $25 \%, 50 \%$, or $75 \%$ of the luminance of the white background. It was found that the contrast-reduced gray ring was less effective in masking a black disk than was its incomplete counterpart.

Arand and Dember (1974) attempted to extend the relationship found by Sherrick and Dember (1968) by again using masking rings having $40 \%$ of the complete ring contour, but with the remaining contour divided into $2,4,6,8,16$, or 32 equal-sized black segments. It was expected that the Sherrick and Dember (1968) finding of an increase in masking effectiveness with increases in ring segments would reach asymptote at some value of ring segments greater than 8 . Beyond that point it was expected that the limits of visual acuity would transform the incomplete ring into one that

Requests for reprints should be sent to Dr. William N. Dember, Department of Psychology, University of Cincinnati, Cincinnati, Ohio 45221. functioned like a complete but contrast-reduced gray ring, which, according to Dember, Colina, and Sherrick (1972), would have reduced effectiveness. The results did indeed demonstrate that the relationship found by Sherrick and Dember (1968) reaches asymptote between 8 and 16 black ring segments. However, the second expectation of the Arand and Dember (1974) study was not supported. Between 16 and 32 black ring segments, masking did not decrease.

The failure of that study to find a decrease in masking effectiveness with increases in masking ring segments may have been due to the fact that the number of masking ring segments used was not sufficiently large. The present study attempted to employ masking rings with a large enough number of segments to allow for an adequate test of the hypothesis of an inverted U-shaped relation between mask effectiveness and number of masking ring segments.

\section{METHOD}

\section{Subjects}

Four male and four female paid college students with normal or corrected-to-normal vision served as subjects. The subjects were trained on all stimuli used in the experiment, receiving 500 trials on each of 2 days before collection of the experimental data.

\section{Stimuli}

The stimuli were black and white photographs on white index cards. The target stimulus was always a solid black disk. There were two sets of masking rings. One set consisted of rings having $40 \%$ of the contour of a complete ring, which was then divided into $2,4,8,16,32,56$, or 64 equal-sized segments and distributed around the ring (alternated with an equal number of white segments). A second set of rings was similar to the first, except that this set had $50 \%$ of the total ring contour of a complete ring. Each mask stimulus consisted of two identical rings horizontally arranged on the white cards. In addition, a complete black ring and a no-ring condition were also used.

The diameter of the disk, as well as the inner diameter of the rings, measured $23.5 \mathrm{~min}$ of arc; the outer diameter of the rings measured $47 \mathrm{~min}$ of arc, and the distance between the centers of each pair of rings was $1 \mathrm{deg} 19 \mathrm{~min}$ of arc. 


\section{Apparatus and Procedure}

The stimuli were presented tachistoscopically (Scientific Prototype, Model GX). The target duration was set at $15 \mathrm{msec}$, while the mask duration was set at $55 \mathrm{msec}$. There was no interstimulus interval. The luminance of all fields, including pre- and postadapting fields, was $25 \mathrm{fL}$. Viewing was monocular. Fixation was guided by two small black dots in the adapting field, one just above each of the loci of the two masking rings.

All subjects were adapted to the fixation field for $5 \mathrm{~min}$ before the start of each session. Each subject received each of the 20 mask conditions in blocks of 25 trials, during each of the four test sessions. At the end of the experiment, all subjects had received 100 trials in each condition. The order of presentation of the mask conditions was randomized independently for each subject.

On each trial the experimenter placed the disk in one of the two rings, the specific location being random. In the no-mask condition, the disk was also randomly placed in one of the two positions it would have occupied if rings had been present. On a signal from the experimenter, the subject pushed a button initiating each trial and then indicated, by saying "right" or "left," in which of the two positions the target appeared, guessing if necessary.

\section{RESULTS AND DISCUSSION}

The percent correct detection was computed for each subject for each stimulus condition. The mean values for the solid and no-mask control conditions were $48 \%$ and $99 \%$, respectively. Data from these control

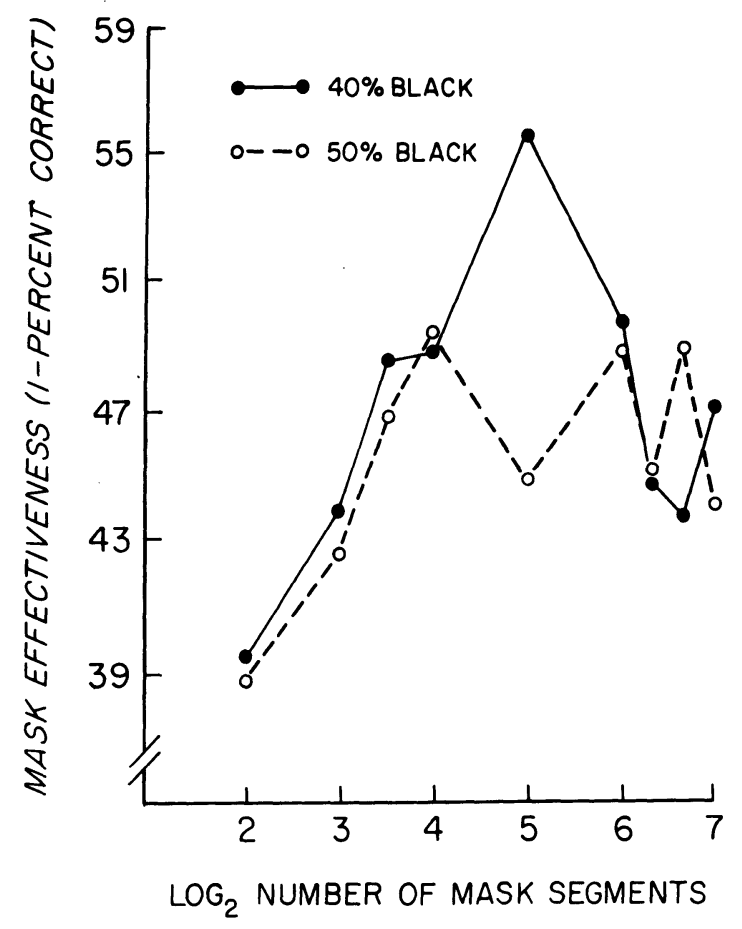

Figure 1. Mask effectiveness as a function of number of black segments in the masking stimulus. The parameter is the percentage of black contour in the masking figure compared with a complete black ring. conditions were not used in the statistical analysis of the results. A trend analysis on the data for the segmented mask conditions showed significant quadratic $(\mathrm{t}=3.31, \mathrm{df}=7, \mathrm{p}<.05)$ and cubic $(\mathrm{t}=3.12$, $\mathrm{df}=7$, $p<.05)$ trends for the overall effect of mask segments. Figure 1 illustrates the results.

In general the data show that an inverted U-shaped relation does hold, as hypothesized, between mask effectiveness and the number of segments in the masking ring. The only notable departure from the relation arises from the 32-segmented mask in the 50\% black condition, which for some unknown reason seems spuriously low.

Though the present experiment was not designed to test any particular theoretical model, it may have some implications for current theories of masking. Thus, the fact that this experiment and previous studies have shown spatial frequency of the mask stimuli to be important in masking is consistent with the theories of masking recently proposed by Breitmeyer and Ganz (1976) and Weisstein, Ozog, and Szoc (1975). In those theories, a spatial frequency analysis of stimuli, performed by the visual system, is postulated to be directly implicated in metacontrast masking. However, while those theories stress the importance of the spatial frequency of the stimuli, the processes they invoke do not seem to be able to account for the present data. According to those models, it is the degree of overlap between the spatial frequency spectrum of the target and mask which determines the amount of masking that will occur. But that expectation is contradicted by the present finding that, for the set of masks having up to about 16 black segments, those with higher spatial frequencies are more effective masks of a low spatial frequency target (solid black) than are the lower spatial frequency masks. Moreover, if the masks with 56 and 64 black segments can be assumed to lie beyond the limits of visual acuity, they should operate as though they were low spatial frequency gray rings, which according to the above theories should result in increased masking of a low spatial frequency target. However, the data show that these stimuli serve as relatively ineffective masks. In essence, then, if we have properly characterized our masking stimuli, the relationship between mask effectiveness and number of segments in the masking figure should have followed a U-shaped, rather than the obtained inverted U-shaped, form.

Of course, our masking stimuli are quite different in structure from the linearly arrayed gratings typically employed in research addressed at the hypothesized spatial frequency analysis. Thus, characterizing our stimuli as being of low or high spatial frequency may be inappropriate. If so, the task still remains for masking models to accommodate results of the sort presented here, which are quite orderly, and hence in need of explanation. 


\section{REFERENCES}

Arand, D., \& Dember, W. N. Masking effectiveness and number of segments in the masking ring. Bulletin of the Psychonomic Society, 1974, 3, 127-128.

BreItMeYER, B., \& GANZ, L. Implications of sustained and transient channels for theories of visual pattern masking, saccadic suppression and information processing. Psychological Review, 1976, 83, 1-36.

Dember, W. N., Colina, T. L., \& Sherrick, M. F. Amount vs contrast of masking figure contour in visual backward masking. Psychonomic Science, 1972, 27, 95-96.
Sherrick, M. F., \& Dember, W. N. Configurational factors in visual backward masking. Proceedings of the 76th Annual Convention of the American Psychological Association, 1968, 3, 111-112.

Weisstein, N., Ozog, G., \& Szoc, R. A comparison and elaboration of two models of metacontrast. Psychological Review, 1975, 82, 325-343.

WERnER, H. Studies on contour: I. Qualitative analyses. American Journal of Psychology, 1935, 47, 40-64.

(Received for publication December 31, 1976.) 